\title{
FOXF2-Mediated IncRNA LINC02532 Promotes Gastric Cancer Cell Migration and Invasion By Decreasing SOX7 mRNA Stability
}

\section{Cheng Zhang}

China Medical University https://orcid.org/0000-0001-5317-8775

Chun-Dong Zhang

China Medical University

Jun-Peng Pei

China Medical University

Yong-Zhi Li

China Medical University

Maimaititusun Yusupu

China Medical University

Dong-Qiu Dai ( $\nabla$ daidq63@163.com )

China Medical University

\section{Research Article}

Keywords: gastric cancer, LINC02532, migration and invasion, FOXF2, SOX7, mRNA stability

Posted Date: December 1st, 2021

DOI: https://doi.org/10.21203/rs.3.rs-1107389/v1

License: (9) (i) This work is licensed under a Creative Commons Attribution 4.0 International License.

Read Full License 


\section{Abstract \\ Background}

LncRNAs are known to play a crucial role in the initiation and progression of human diseases, especially cancers. Our previous study demonstrated that dysregulation of LINC02532 facilitated the malignant phenotype of gastric cancer (GC). However, the potential molecular mechanisms regarding the upstream and downstream regulation of LINC02532 in GC progression remain unclear.

\section{Methods}

RNA-Seq and clinical data from public databases were used for gene expression and clinical analyses. The subcellular location of LINC02532 was predicted by the bioinformatics tools and further validated by the RNA-Fluorescence in situ hybridization (FISH) assay. The effect of FOXF2/LINC02532/SOX7 axis in GC cell migration and invasion was evaluated using in vitro and in vivo assays. The transcriptional regulation role of FOXF2 and the mRNA stability of SOX7 were explored by dual-luciferase reporter assay and Actinomycin-D drug assay.

\section{Results}

We found that high LINC02532 expression was associated with poor prognosis of GC. Furthermore, a Cox regression model indicated that LINC02532 was an independent prognostic factor for GC patients. Using in vitro and in vivo assays, we found that LINC02532 promoted GC cell migration and invasion, as well as tumour growth and metastasis in nude mice. Mechanistically, LINC02532 decreased SOX7 mRNA stability by binding to its $3^{\prime} U T R$, resulting in reduced SOX7 expression. In addition, FOXF2 was identified as a transcriptional factor of LINC02532 and was shown to repress LINC02532 expression by negative transcriptional regulation.

\section{Conclusions}

Together, these findings show that LINC02532 promotes GC progression through epithelialmesenchymal transition (EMT). Cross-talk between the FOXF2/LINC02532/SOX7 axis may provide a novel target for the treatment and prognostic prediction of GC.

\section{Background}

Gastric cancer (GC) is one of the most common malignant tumours of the digestive tract. In 2018, the International Agency for Research on Cancer (GLOBOCAN) reported 1,033,701 new cases of GC and 782,685 GC-related deaths worldwide ${ }^{1}$. GC ranked second in morbidity and mortality rates of all cancers 
in China in $2015^{2}$. Furthermore, China accounts for more than $40 \%$ of GC cases worldwide ${ }^{1,3}$. Due to advances in detection methods as well as a gradual improvement in health awareness, the incidence of GC is increasing in younger patients. Furthermore, most patients are already at an advanced stage when diagnosed ${ }^{4}$.

The Human Genome Project found that only $1 \%$ of the genome encodes protein-coding genes ${ }^{5}$. The transcribed products of the remaining $99 \%$ of the genome are called non-coding transcripts, and include housekeeping non-coding RNA (such as ribosomal, transfer and small nuclear RNA) and regulatory noncoding RNA (such as microRNA and long-sequence non-coding RNA (IncRNA)) ${ }^{6}$. Although IncRNA, with a length of more than 200 nucleotides, has no protein coding function, it plays a vital role in the occurrence and development of human cancers at epigenetic ${ }^{7}$, transcriptional ${ }^{8}$ and post-transcriptional levels ${ }^{9}$. Our previous study found that LINC02532 was significantly upregulated in GC cell lines and tissues, and that the silencing of LINC02532 expression could suppress GC cell proliferation, migration, and invasion ${ }^{10}$. However, the precise function of LINC02532 in the initiation and development of GC remains unclear.

In the present study, we show that LINC02532 decreases SOX7 mRNA stability by binding to its 3'UTR. In addition, we identify forkhead box F2 (FOXF2) as a transcriptional suppressor of LINC02532 expression. Taken together, these results identify the FOXF2/LINC02532/SOX7 axis as a vital mediator in the migration and invasion of GC cells.

\section{Methods}

\section{Database source}

The RNA-Seq and clinical data of stomach cancer patients from UCSC Xena datasets ${ }^{11}$, RNA-Seq data of multiple human normal tissues from The Genotype-Tissue Expression (GTEx) project ${ }^{12}$, and protein staining data from The Human Protein Atlas ${ }^{13}$ were downloaded and used for further analyses.

\section{Patients and tissues}

Thirty GC tissue specimens were obtained from patients who underwent stomach surgery at the Fourth Affiliated Hospital of China Medical University (Shenyang, China). All specimens were confirmed by histopathological examination and were stored at $-80^{\circ} \mathrm{C}$ immediately after surgical resection. All patients provided written informed consent and the study protocol was approved by the Research Ethics Committee of the Fourth Affiliated Hospital of China Medical University.

\section{Cell Culture and quantitative real-time RT-PCR}

Detailed protocols were performed as described in our previous study ${ }^{10}$. Specific primers for qRT-PCR are shown in Supplementary Table 1.

\section{Cell transfection}


The specific siRNA against LINC02532 (Si-LINC02532 \#1 and \#2), FOXF2 (Si-FOXF2 \#1 and \#2), SOX7 (Si-SOX7), and the corresponding negative control (Si-NC), together with the pcDNA3.1 vector targeting LINC02532, FOXF2, SOX7, and the corresponding empty vector, were constructed by RiboBio (Guangzhou, China). Lipofectamine 3000 (Invitrogen, Carlsbad, CA, USA) was utilized to transfect these vectors into SGC-7901, MGC-803 and HEK293T cells. The primer sequences are shown in Supplementary Table 1.

\section{RNA-Fluorescence in situ hybridization (FISH) and protein coding potential}

The subcellular location of LINC02532 was first predicted by the bioinformatics tools, IncLocator ${ }^{14}$ and iLoc-LncRNA ${ }^{15}$. The FISH assay was carried out to confirm the subcellular location of LINC02532 using the FISH Kit (GenePharma, Shanghai, China) with Cy3-labeled LINC02532 probes according to the manufacturer's protocol (Supplementary Table 1). The protein coding potential of LINC02532 was predicted by LNCipedia version $5.2^{16}$, which integrates different algorithms from PRoteomics IDEntifications (PRIDE) ${ }^{17}$, translation initiation sites ${ }^{18}$, PhyloCSF $^{19}$, Coding Potential Assessing Tool (CPAT) ${ }^{20}$ and small ORFs ${ }^{21}$.

\section{Cell migration and invasion assays}

The Transwell assays were performed as described in our previous study ${ }^{10}$.

\section{Western blot analysis}

Samples were lysed using the RIPA buffer (Solarbio, Beijing, China). Equivalent amounts of total protein were separated on $8 \%$ SDS-PAGE gels and transferred to PVDF membranes (Millipore, Billerica, MA, USA). The PVDF membranes were blocked with $5 \%$ skim milk for $2 \mathrm{~h}$ by gentle shaking. Then, the primary antibodies were incubated at $4^{\circ} \mathrm{C}$ overnight according to the following dilution ratios: E-cadherin (1:200; Abcam, Cambridge, MA, USA), N-cadherin (1:2000; Abcam), Vimentin (1:1000; Abcam), Slug (1:2000; Abcam), SOX7 (1:1000; Abcam), and $\beta$-actin (1:2500; Abcam). The membranes were washed the following day and incubated with horseradish peroxidase-conjugated secondary antibody (goat antirabbit, 1:5000; Wanleibio). Finally, enhanced chemiluminescence (ECL) substrate was used to analyse the signals with a chemiluminescence detection system (Tanon, Shanghai, China).

\section{Prediction of target binding sites and dual-luciferase reporter assay}

The sequence from -2000 to +100 bp relative to the transcription start site (TSS) of LINC02532 was regarded as the promoter region. The transcriptional binding sites of FOXF2 on the gene promoter region of LINC02532 were predicted by the JASPAR CORE database ${ }^{22}$. The RNA-RNA interaction binding bites between LINC02532 and the 3'UTR region of SOX7 mRNA were obtained using the IntaRNA program ${ }^{23}$.

Further verification of target binding sites was conducted using the dual-luciferase reporter assay. For the LINC02532-SOX7 3'UTR luciferase reporter assay, sequences containing the predicted RNA-RNA interaction binding bites (pGL3-SOX7 3'UTR-WT/Mut) were inserted into the pGL3-basic firefly luciferase 
reporter (Promega, Madison, WI, USA), then co-transfected with pcDNA3.1-LINC02532 or empty vector into HEK293T and MGC-803 cells. For the LINC02532 promoter luciferase reporter assay, sequences containing the predicted FOXF2 binding sites (pGL3-LINC02532-WT/Mut) were also inserted into the pGL3-basic firefly luciferase reporter, then co-transfected with pcDNA3.1-FOXF2 or empty vector into HEK293T and MGC-803 cells. After $24 \mathrm{~h}$ of transfection, relative luciferase activities were measured using the Dual-Luciferase Reporter Assay System (Promega) and normalized to the control Renilla.

\section{mRNA stability assay}

SGC-7901 and MGC-803 cells $\left(1 \times 10^{6}\right)$ transfected with Si-LINC02532 \#1, \#2 and Si-NC were seeded into six-well plates. Then, $5 \mu \mathrm{g} / \mathrm{ml}$ of Actinomycin-D (ActD, MedChem Express) was added to the medium to block the de novo RNA synthesis. After treatment for 3,6 , and $9 \mathrm{~h}$, cells were harvested and total RNA was isolated for measuring SOX7 mRNA levels by qRT-PCR.

\section{Subcutaneous xenograft models}

In vivo assays were conducted using BALB/C nude mice (female, 4 weeks old). A total of $1 \times 10^{6} \mathrm{MGC}$ 803 cells transfected with LINC02532 overexpression or negative control were injected into the dorsal right flank, respectively ( $n=4$ per group). Tumour diameter and volume were measured and calculated every 3 days for 3 weeks. All experiments were approved by the Research Ethics Committee of the Fourth Affiliated Hospital of China Medical University and carried out based on the guidelines of the Institutional Animal Care and Use Committee.

\section{Lung metastasis assay}

For the lung metastasis model, the corresponding GC cells were injected into the tail veins of nude mice. Then, a total of 10 nude mice were randomly separated into two groups (5 mice in each group). Six weeks after the injection, the nude mice were euthanatized. Next, all the lung tissues were dissociated and metastatic nodules were counted.

\section{Gene set enrichment analysis (GSEA)}

GC patients from The Cancer Genome Atlas (TCGA) were divided into high or low expression groups according to the median value of LINC02532 expression. GSEA was performed on the RNA-Seq data to explore differences in biological pathways between high and low LINC02532 expression groups.

\section{Statistical analysis}

SPSS version 20.0 (SPSS, Inc., Chicago, IL, USA) and Prism version 7.0 (GraphPad Software, Inc., La Jolla, CA, USA) statistical software were used for statistical analysis. The difference between two groups was compared with two-tailed Student's $t$-test, and data were presented as the mean \pm standard deviation. A chi-squared test was used to compare the clinicopathologic characteristics of GC patients and LINC02532 expression level. Pearson correlation analysis was used to assess gene expression 
relationships. The optimal cut-off value for dividing patients into high or low LINC02532 expression groups was calculated using X-tile software ${ }^{24}$. The univariate and multivariate Cox regression analysis were carried out to assess the prognostic value of LINC02532 expression. A nomogram for the 1-, 2-, and 3-year OS prediction was constructed using LINC02532 expression and clinical factors $(p<0.2$ was used to select variables to construct the model). Kaplan-Meier survival curve and log-rank tests were performed to evaluate the overall survival in relation to LINC02532 expression. $\mathrm{P}<0.05$ indicated a significant difference.

\section{Results}

\section{LINC02532 is located in the cytoplasm and is significantly upregulated in GC}

In our previous study ${ }^{10}$, we identified differentially expressed IncRNAs in GC using RNA-Seq data from the TCGA project, and showed using qRT-PCR that LINC02532 was significantly upregulated in GC cell lines and tissues. In order to examine the distribution and expression of LINC02532 in human organs, we obtained and analysed the global RNA expression of multiple human tissues from the GTEx project. We found that LINC02532 was widely expressed in various human tissues, including the kidney, liver, pancreas, and stomach (Figure 1A). Furthermore, similar LINC02532 expression levels were observed in multiple normal tissues independent of sex (Figure 1B). According to the InCAR database ${ }^{25}$, LINC02532 is a non-protein coding RNA located on chromosome 6 (6q21) (Figure 1C). Numerous protein-coding projects agree that LINC02532 has no protein-coding function (Table 1). To investigate the potential molecular role of LINC02532, we used bio-informatics to determine its subcellular location. Our findings indicated that LINC02532 would be predominantly located in the cytoplasm (Figure 1D). RNA-FISH analysis confirmed that LINC02532 was mainly located in the cytoplasm of SGC-7901 and MGC-803 cells (Figure 1E). These findings suggest that LINC02532 may play an oncogenic role in the cytoplasm.

Table 1

Protein coding potential of LINC02532.

\begin{tabular}{|lll|}
\hline Metric & Raw result & Interpretation \\
\hline PRIDE reprocessing 2.0 [36] & 0 & Non-coding \\
\hline Lee translation initiation sites [37] & NA & Non-coding \\
\hline PhyloCSF score [38] & -88.8068 & Non-coding \\
\hline CPAT coding probability [39] & $2.69 \%$ & Non-coding \\
\hline Bazzini small ORFs [40] & 0 & Non-coding \\
\hline NA, not available. & & \\
\hline
\end{tabular}


A total of 329 GC patients with full clinicopathologic data from the TCGA project were included in the present study. First, the optimal cut-off value for dividing patients into high or low LINC02532 expression groups was calculated using the X-tile software program. The optimal cut-off expression value was 0.2 (log2 transformed) (Figure 2A). Kaplan-Meier survival analysis indicated that GC patients with higher LINC02532 expression had a significantly shorter overall survival (OS) time than those with lower LINC02532 expression (Figure 2B). Chi-squared association analysis showed that higher LINC02532 expression was significantly correlated with age and $\mathrm{M}$ stage. However, there was no significant relationship between LINC02532 expression and gender, T/N stage and pathological stage (Table 2). Univariate and multivariate Cox regression analyses revealed that high LINC02532 expression was an independent prognostic factor for GC patients (Figure 2C). A nomogram for the 1-, 2-, and 3-year OS prediction was constructed using LINC02532 expression and clinical factors. The concordance index was 0.66 and had a satisfactory predictive ability (Figure 2D). These findings suggest that LINC02532 expression has vital clinical significance and could be used as a prognostic biomarker for GC patients. 
Table 2

Association between LINC02532 expression and clinicopathological characteristics of GC patients $(n=329)$.

\begin{tabular}{|c|c|c|c|c|}
\hline \multirow[t]{2}{*}{ Clinical features } & \multicolumn{2}{|c|}{ LINC02532 expression } & \multirow[t]{2}{*}{ Number $(n=329)$} & \multirow[t]{2}{*}{$P$ value } \\
\hline & Low $(n, \%)$ & High (n,\%) & & \\
\hline \multicolumn{5}{|l|}{ Age } \\
\hline$<60$ & $28(8.5)$ & $73(22.2)$ & 101 & \multirow[t]{2}{*}{$0.001^{* \star}$} \\
\hline$\geq 60$ & $110(33.4)$ & $118(35.9)$ & 228 & \\
\hline \multicolumn{5}{|l|}{ Gender } \\
\hline Male & $85(25.8)$ & $124(37.7)$ & 209 & \multirow[t]{2}{*}{0.536} \\
\hline Female & $53(16.1)$ & $67(20.4)$ & 120 & \\
\hline \multicolumn{5}{|l|}{ T stage } \\
\hline $\mathrm{T} 1+\mathrm{T} 2$ & $39(11.9)$ & $42(12.8)$ & 81 & \multirow[t]{2}{*}{0.193} \\
\hline $\mathrm{T} 3+\mathrm{T} 4$ & $99(30.1)$ & $149(45.2)$ & 248 & \\
\hline \multicolumn{5}{|l|}{$\mathrm{N}$ stage } \\
\hline NO & $49(14.9)$ & $52(15.8)$ & 101 & \multirow[t]{2}{*}{0.108} \\
\hline N1-3 & $89(27.1)$ & $139(42.2)$ & 228 & \\
\hline \multicolumn{5}{|l|}{ M stage } \\
\hline M0 & $129(39.2)$ & $164(49.8)$ & 293 & \multirow[t]{2}{*}{$0.029 *$} \\
\hline M1 & $9(2.8)$ & $27(8.2)$ & 36 & \\
\hline \multicolumn{5}{|l|}{ Pathologic stage } \\
\hline Stage $\mid+\|$ & $70(21.3)$ & $80(24.3)$ & 150 & \multirow[t]{2}{*}{0.112} \\
\hline Stage III+IV & $68(20.7)$ & $111(33.7)$ & 179 & \\
\hline
\end{tabular}

LINC02532 promotes GC cell migration and invasion in vitro and in vivo

We explored the biological function of LINC02532 in GC cells. First, we found that LINC02532 was overexpressed in SGC-7901 and MGC-803 cells transfected with the pcDNA-LINC02532 (Figure 3A). Similar to previous findings ${ }^{10}$, LINC02532 overexpression significantly promoted GC cell migration and invasion in the Transwell assay (Figure 3B). Next, we investigated the potential role of LINC02532 in the epithelial-mesenchymal transition (EMT) process. The mRNA and protein levels of an epithelial marker (Ecadherin) and mesenchymal markers (N-cadherin, VIM, and SLUG) were measured by qRT-PCR and 
western blotting. Silencing LINC02532 expression significantly increased the expression of E-cadherin and inhibited the expression of N-cadherin, VIM, and SLUG in GC cells (Figure 3C, D). Furthermore, in vivo, LINC02532 overexpression significantly increased tumour growth rate and tumour weight in nude mice (Figure 3E). The average tumour weight of the LINC02532 overexpression group was increased by 1.57fold compared to the negative control group $(0.308 \pm 0.066 \mathrm{~g}$ vs. $0.120 \pm 0.031 \mathrm{~g})$. For lung metastasis assay, the LINC02532 overexpression group mice had more metastatic nodules in lung than that in control group ( $43.40 \pm 9.84$ vs. $12.00 \pm 3.87$ ). Taken together, these results suggest that LINC02532 functions as an oncogene in GC cells.

\section{Linc02532 Decreases Sox7 Expression Via Regulation Of Mrna Stability}

After establishing the oncogenic role of LINC02532, we further explored the molecular mechanisms by which LINC02532 promotes GC cell migration and invasion. Gene Set Enrichment Analysis (GSEA) of the RNA-Seq expression matrix data found a significant positive correlation between high levels of LINC02532 expression and the KEGG RNA degradation pathway (Figure 4A). Since LINC02532 is predominantly located in the cytoplasm, we speculated that LINC02532 may be involved in the regulation of mRNA stability.

Based on the Human Protein Atlas (HPA) pathology data, we found a moderate level of SOX7 protein expression in normal glandular stomach, however, GC tissues displayed moderate (2/12), low (5/12) or undetectable (5/12) levels of SOX7 (Supplementary Figure 1). Using qRT-PCR, we demonstrated that SOX7 mRNA levels were significantly down-regulated in GC tissues compared to the corresponding normal tissues (Figure 4B). Next, we found that SOX7 expression was negatively correlated with LINC02532 expression (Pearson $r=-0.45, p=0.0014$, Figure 4C). Finally, we measured the mRNA and protein levels of SOX7 in SGC-7901 and MGC-803 cells treated with control siRNA (si-NC) or a LINC02532specific siRNA (si-LINC02532) by qRT-PCR and western blotting. Compared to the control si-NC group, SOX7 mRNA and protein expression were both up-regulated in the si-LINC02532 group (Figure 4D), further highlighting the relationship between LINC02532 and SOX7.

Using RNA-RNA interaction prediction software, we identified binding sites between LINC02532 and the SOX7 mRNA 3'UTR region (Supplementary Figure 2). We constructed a luciferase reporter plasmid containing the wild-type or mutated SOX7 mRNA 3'UTR region (SOX7 3'UTR-WT or SOX7 3'UTR-Mut), and then transfected the plasmids into HEK293T and MGC-803 cells with pcDNA-LINC02532 or an empty vector. The relative luciferase activity of SOX7 3'UTR-WT in PCDNA-LINC02532 was significantly decreased, however, no difference was found when the binding sites were mutated (Figure 4E). These findings indicate that LINC02532 negatively regulates SOX7 expression by binding to its $3^{\prime} U T R$ region. To further assess whether LINC02532 affected SOX7 mRNA degradation, we measured SOX7 mRNA levels in GC cells treated with the RNA synthesis inhibitor Actinomycin D (ActD) in the presence (si-NC) or absence (si-LINC02532) of LINC02532. Compared to the control si-NC group, SOX7 mRNA stability was 
increased when LINC02532 expression was silenced, suggesting that LINC02532 inhibited SOX7 expression by decreasing its mRNA stability (Figure 4F). Next, the effect of the LINC02532-SOX7 axis on the migration and invasion of GC cells was examined using a Transwell assay. We found that silencing SOX7 expression promoted GC cell migration and invasion, and that reducing LINC02532 expression with si-LINC02532 partially rescues this effect (Figure 4G). Taken together, these results suggest that LINC02532 promotes GC cell migration and invasion by decreasing SOX7 mRNA stability.

\section{FOXF2 mediates GC cell migration and invasion by negatively regulating LINC02532 transcription}

Next, we explored the potential upstream mechanism regulating LINC02532 in GC. The GSEA data showed that high LINC02532 expression was significantly negatively correlated with the KEGG basal transcription factors pathway (Figure 5A). FOXF2, a transcription factor of the forkhead box family, has been previously reported to act as a tumour suppressor in multiple cancers including breast, stomach, and liver ${ }^{26-28}$. Using the JASPAR CORE database, we found that FOXF2 possesses binding sites on the LINC02532 promoter region. Thus, we speculated that LINC02532 may be regulated by FOXF2.

Using qRT-PCR, we found that FOXF2 was significantly down-regulated in $30 \mathrm{GC}$ tissues and that its expression level was negatively correlated with LINC02532 (Figure 5B, C). In addition, LINC02532 expression was significantly up-regulated after silencing FOXF2 expression in SGC-7901 and MGC-803 cells (Figure 5D). A conserved FOXF2-binding site ( 5 ' - CACATGATAATCAAT - 3 ') located at -469 to -456 bp from the transcription start site (TSS) of LINC02532 was utilized for transcription factor binding verification (Figure $5 \mathrm{E}$ ). The dual-luciferase reporter assay showed that the relative luciferase activity of LINC02532-WT (containing the FOXF2-binding site) in pCDNA-FOXF2 was significantly decreased. In contrast, no difference was found when the FOXF2-binding site was mutated (Figure 5F). These findings suggest that FOXF2 negatively regulates LINC02532 expression through transcriptional suppression. Using a Transwell assay, we showed that silencing LINC02532 expression inhibited GC cell migration and invasion, and that the knockdown of FOXF2 using siRNA (si-FOXF2) partially rescues this effect (Figure $5 G)$.

In conclusion, we found that the down-regulation of FOXF2 results in increased LINC02532 transcription, and that LINC02532 inhibits SOX7 expression by decreasing SOX7 mRNA stability in GC. Thus, we propose a novel FOXF2/LINC02532/SOX7 axis in the migration and invasion of GC cells (Figure 6).

\section{Discussion}

SOX7, located on the human chromosome 8 p23.1, is a member of the SOXF gene subfamily ${ }^{29}$. As a developmental regulator, SOX7 was reported to be involved in hematopoietic development through the regulation of vascular endothelial cadherin expression ${ }^{30}$. It also suppressed cellular proliferation and induced apoptosis in multiple cancers, including breast, colon, kidney, and gastric cancer ${ }^{31-34}$. However, the molecular mechanism regulating SOX7 in GC remains unclear. In our previous study, we demonstrated that LINC02532 was up-regulated in GC and had an oncogenic role in the development and 
progression of GC ${ }^{10}$. LINC02532 was shown to facilitate the malignant phenotype of GC in a series of in vitro and in vivo functional experiments. Furthermore, we found that silencing LINC02532 significantly increased E-cadherin expression while inhibiting N-cadherin, VIM and SLUG expression levels in GC cells, indicating that LINC02532 may be involved in the process of EMT. However, it was still unclear whether LINC02532 mediated the downregulation of SOX7.

We performed GSEA on the RNA-Seq data to explore the potential biological pathways in which LINC02532 may be involved. We found a significant positive correlation between LINC02532 and the KEGG RNA degradation pathway. RNA-RNA interaction prediction software suggested that LINC02532 could bind to the SOX7 mRNA 3'UTR region. Yan et al. demonstrated that up-regulation of LINC00470 promoted GC cell proliferation, migration, and invasion via degradation of PTEN mRNA stability ${ }^{35}$. Since LINC02532 was predominantly located in the cytoplasm, we hypothesized that LINC02532 may inhibit SOX7 expression via regulation of mRNA stability. Pearson expression relationship analysis indicated that SOX7 was negatively correlated with LINC02532 expression. Furthermore, the mRNA and protein level of SOX7 in SGC-7901 and MGC-803 cells were both increased after LINC02532 expression was silenced. The dual-luciferase reporter assay further confirmed that LINC02532 bound to the SOX7 mRNA 3'UTR region. Thus, our findings show that LINC02532 promotes GC cell migration and invasion by decreasing SOX7 mRNA stability.

As known to all, mounting evidences have showed that IncRNAs act as competing endogenous RNAs (ceRNAs) to participate in mRNA expression regulation in human cancers ${ }^{36}$. However, the mRNA stability dysregulation mediated by IncRNAs was still poorly understood. Although the downstream regulatory mechanisms of IncRNAs are well documented, the upstream molecular mechanisms which regulate IncRNAs require further elucidation. FOXF2, a member of the Fox family of forkhead box transcription factors, is characterized by the presence of a highly conserved winged helix DNA binding domain ${ }^{37}$. Recent studies have found that FOXF2 is dysregulated in multiple human cancers and is involved in cancer cell proliferation, invasion and metastasis ${ }^{28,38-40}$. Mechanistically, FOXF2 could regulate gene expression through transcriptional suppression. Kundu et al. ${ }^{40}$ found that FOXF2 transcriptionally repressed E-cadherin and miR-200, and was involved in lung cancer invasion and metastasis. In basal-like breast cancer, FOXF2 inhibited the EMT and multidrug resistance via negative regulation of FOXC2 expression ${ }^{41}$. Milewski et al. demonstrated that FOXF2 directly bound to the promoter region of p21 and repressed its transcriptional activity in rhabdomyosarcoma 42 .

The GSEA data also showed that high LINC02532 expression was significantly negatively correlated with the KEGG basal transcription factors pathway. Using the JASPAR CORE database, we found that FOXF2 possesses binding sites on the LINC02532 promoter region. Thus, we speculated that FOXF2 may transcriptionally regulate LINC02532 expression. Interestingly, LINC02532 expression was significantly up-regulated after FOXF2 expression was silenced in SGC-7901 and MGC-803 cells. The dual-luciferase reporter assay confirmed that FOXF2 could bind on the promoter region located at -469 to $-456 \mathrm{bp}$ from the TSS of LINC0253. Furthermore, cell functional rescue experiments revealed that silencing LINC02532 
inhibited GC cell migration and invasion, whereas si-FOXF2 partially rescued the effect induced by LINC02532 inhibition. In conclusion, our studies show that FOXF2 suppresses GC cell migration and invasion by negatively regulating LINC02532 transcription.

\section{Conclusions}

The present study reveals that LINC02532 has a role in facilitating the malignant phenotype of GC cells both in vitro and in vivo. Clinical significance analysis indicates that LINC02532 is an independent prognostic factor for GC patients. Mechanistically, we found that LINC02532 reduces the mRNA stability of SOX7 through binding to its 3'UTR. In addition, downregulation of FOXF2 results in decreased transcriptional suppression of LINC02532. Thus, our findings identify the novel FOXF2-LINC02532-SOX7 axis as a vital mediator in the migration and invasion of GC cells.

\section{List Of Abbreviations}

GC: gastric cancer

LncRNA: long-sequence non-coding RNA

FOXF2: forkhead box F2

GTEx: Genotype-Tissue Expression

FISH: Fluorescence in situ hybridization

GSEA: Gene set enrichment analysis

EMT: Epithelial-mesenchymal transition

HPA: Human Protein Atlas

\section{Declarations}

\section{Ethics approval and consent to participate}

All patients in present study provided written informed consent and the study protocol was approved by the Research Ethics Committee of the Fourth Affiliated Hospital of China Medical University. The animal experiment was approved by the Research Ethics Committee of the Fourth Affiliated Hospital of China Medical University and carried out based on the guidelines of the Institutional Animal Care and Use Committee.

\section{Consent for publication}

Not applicable. 


\section{Data availability statements}

The datasets used and/or analysed during the current study are available from the corresponding author on reasonable request.

\section{Conflicts of interest}

The authors declare no competing interest.

\section{Funding}

This study was supported by the National Natural Science Foundation of China (No. 81972322) and the China Scholarship Council (No. 201908050148).

\section{Author Contributions}

Dai DQ, Zhang C and Zhang CD designed the study; Zhang C, Pei JP, Li YZ and Yusupu M performed data processing and experiment analysis; Zhang $\mathrm{C}$ was a major contributor in writing the manuscript. All authors read and approved the final manuscript.

\section{References}

1. Bray F, Ferlay J, Soerjomataram I, Siegel RL, Torre LA and Jemal A (2018). Global cancer statistics 2018: GLOBOCAN estimates of incidence and mortality worldwide for 36 cancers in 185 countries. CA Cancer J Clin 68: 394-424.

2. Chen W, Zheng R, Baade PD, Zhang S, Zeng H, Bray F, Jemal A, Yu XQ and He J (2016). Cancer statistics in China, 2015. CA Cancer J Clin 66: 115-132.

3. Feng RM, Zong YN, Cao SM and Xu RH (2019). Current cancer situation in China: good or bad news from the 2018 Global Cancer Statistics? Cancer Commun (Lond) 39: 22.

4. Zhang XY and Zhang PY (2017). Gastric cancer: somatic genetics as a guide to therapy. J Med Genet 54: 305-312.

5. Schuler GD, Boguski MS, Stewart EA, Stein LD, Gyapay G, Rice K, White RE, Rodriguez-Tome P, Aggarwal A, Bajorek E, et al. (1996). A gene map of the human genome. Science 274: 540-546.

6. Ponting CP, Oliver PL and Reik W (2009). Evolution and functions of long noncoding RNAs. Cell 136: $629-641$.

7. Ntziachristos P, Abdel-Wahab $O$ and Aifantis I (2016). Emerging concepts of epigenetic dysregulation in hematological malignancies. Nat Immunol 17: 1016-1024.

8. Yu Y, Yarrington RM, Chuong EB, Elde NC and Stillman DJ (2016). Disruption of promoter memory by synthesis of a long noncoding RNA. Proc Natl Acad Sci U S A 113: 9575-9580.

9. Yoon JH, Abdelmohsen K and Gorospe M (2013). Posttranscriptional gene regulation by long noncoding RNA. J Mol Biol 425: 3723-3730. 
10. Zhang C, Ma MH, Liang Y, Wu KZ and Dai DQ (2019). Novel long non-coding RNA LINC02532 promotes gastric cancer cell proliferation, migration, and invasion in vitro. World J Gastrointest Oncol 11: $91-101$.

11. Goldman MJ, Craft B, Hastie M, Repecka K, McDade F, Kamath A, Banerjee A, Luo Y, Rogers D, Brooks AN, et al. (2020). Visualizing and interpreting cancer genomics data via the Xena platform. Nat Biotechnol 38: 675-678.

12. Consortium GT (2013). The Genotype-Tissue Expression (GTEx) project. Nat Genet 45: 580-585.

13. Uhlen M, Zhang C, Lee S, Sjostedt E, Fagerberg L, Bidkhori G, Benfeitas R, Arif M, Liu Z, Edfors F, et al. (2017). A pathology atlas of the human cancer transcriptome. Science 357.

14. Cao Z, Pan X, Yang Y, Huang Y and Shen HB (2018). The IncLocator: a subcellular localization predictor for long non-coding RNAs based on a stacked ensemble classifier. Bioinformatics 34 : 2185-2194.

15. Su ZD, Huang Y, Zhang ZY, Zhao YW, Wang D, Chen W, Chou KC and Lin H (2018). iLoc-IncRNA: predict the subcellular location of IncRNAs by incorporating octamer composition into general PseKNC. Bioinformatics 34: 4196-4204.

16. Volders PJ, Anckaert J, Verheggen K, Nuytens J, Martens L, Mestdagh P and Vandesompele J (2019). LNCipedia 5: towards a reference set of human long non-coding RNAs. Nucleic Acids Res 47: D135D139.

17. Vizcaino JA, Cote RG, Csordas A, Dianes JA, Fabregat A, Foster JM, Griss J, Alpi E, Birim M, Contell J, et al. (2013). The PRoteomics IDEntifications (PRIDE) database and associated tools: status in 2013. Nucleic Acids Res 41: D1063-1069.

18. Lee S, Liu B, Lee S, Huang SX, Shen B and Qian SB (2012). Global mapping of translation initiation sites in mammalian cells at single-nucleotide resolution. Proc Natl Acad Sci U S A 109: E2424-2432.

19. Lin MF, Jungreis I and Kellis M (2011). PhyloCSF: a comparative genomics method to distinguish protein coding and non-coding regions. Bioinformatics 27: i275-282.

20. Wang L, Park HJ, Dasari S, Wang S, Kocher JP and Li W (2013). CPAT: Coding-Potential Assessment Tool using an alignment-free logistic regression model. Nucleic Acids Res 41: e74.

21. Bazzini AA, Johnstone TG, Christiano R, Mackowiak SD, Obermayer B, Fleming ES, Vejnar CE, Lee MT, Rajewsky N, Walther TC, et al. (2014). Identification of small ORFs in vertebrates using ribosome footprinting and evolutionary conservation. EMBO J 33: 981-993.

22. Fornes O, Castro-Mondragon JA, Khan A, van der Lee R, Zhang X, Richmond PA, Modi BP, Correard S, Gheorghe M, Baranasic D, et al. (2020). JASPAR 2020: update of the open-access database of transcription factor binding profiles. Nucleic Acids Res 48: D87-D92.

23. Mann M, Wright PR and Backofen R (2017). IntaRNA 2.0: enhanced and customizable prediction of RNA-RNA interactions. Nucleic Acids Res 45: W435-W439.

24. Camp RL, Dolled-Filhart M and Rimm DL (2004). X-tile: a new bio-informatics tool for biomarker assessment and outcome-based cut-point optimization. Clin Cancer Res 10: 7252-7259. 
25. Zheng Y, Xu Q, Liu M, Hu H, Xie Y, Zuo Z and Ren J (2019). InCAR: A Comprehensive Resource for IncRNAs from Cancer Arrays. Cancer Res 79: 2076-2083.

26. Kong PZ, Yang F, Li L, Li XQ and Feng YM (2013). Decreased FOXF2 mRNA expression indicates early-onset metastasis and poor prognosis for breast cancer patients with histological grade II tumor. PLoS One 8: e61591.

27. Higashimori A, Dong Y, Zhang Y, Kang W, Nakatsu G, Ng SSM, Arakawa T, Sung JJY, Chan FKL and Yu J (2018). Forkhead Box F2 Suppresses Gastric Cancer through a Novel FOXF2-IRF2BPL-betaCatenin Signaling Axis. Cancer Res 78: 1643-1656.

28. Shi Z, Liu J, Yu X, Huang J, Shen S, Zhang Y, Han R, Ge N and Yang Y (2016). Loss of FOXF2 Expression Predicts Poor Prognosis in Hepatocellular Carcinoma Patients. Ann Surg Oncol 23: 211217.

29. Bowles J, Schepers $G$ and Koopman P (2000). Phylogeny of the SOX family of developmental transcription factors based on sequence and structural indicators. Dev Bio/ 227: 239-255.

30. Costa G, Mazan A, Gandillet A, Pearson S, Lacaud G and Kouskoff V (2012). SOX7 regulates the expression of VE-cadherin in the haemogenic endothelium at the onset of haematopoietic development. Development 139: 1587-1598.

31. Wang L, Fan Y, Zhang L, Li L, Kuang G, Luo C, Li C, Xiang T, Tao Q, Zhang Q, et al. (2019). Classic SRY-box protein SOX7 functions as a tumor suppressor regulating WNT signaling and is methylated in renal cell carcinoma. FASEB J 33: 254-263.

32. Yang M, Cui G, Ding M, Yang W, Liu Y, Dai D and Chen L (2016). miR-935 promotes gastric cancer cell proliferation by targeting SOX7. Biomed Pharmacother 79: 153-158.

33. Stovall DB, Wan M, Miller LD, Cao P, Maglic D, Zhang Q, Stampfer MR, Liu W, Xu J and Sui G (2013). The regulation of SOX7 and its tumor suppressive role in breast cancer. Am J Pathol 183: 16451653.

34. Guo L, Zhong D, Lau S, Liu X, Dong XY, Sun X, Yang VW, Vertino PM, Moreno CS, Varma V, et al. (2008). Sox7 Is an independent checkpoint for beta-catenin function in prostate and colon epithelial cells. Mol Cancer Res 6: 1421-1430.

35. Yan J, Huang X, Zhang X, Chen Z, Ye C, Xiang W and Huang Z (2020). LncRNA LINC00470 promotes the degradation of PTEN mRNA to facilitate malignant behavior in gastric cancer cells. Biochem Biophys Res Commun 521: 887-893.

36. Liang Y, Zhang CD, Zhang C and Dai DQ (2020). DLX6-AS1/miR-204-5p/OCT1 positive feedback loop promotes tumor progression and epithelial-mesenchymal transition in gastric cancer. Gastric Cancer 23: 212-227.

37. Hellqvist M, Mahlapuu M, Blixt A, Enerback S and Carlsson P (1998). The human forkhead protein FREAC-2 contains two functionally redundant activation domains and interacts with TBP and TFIIB. J Biol Chem 273: 23335-23343.

38. Myatt SS and Lam EW (2007). The emerging roles of forkhead box (Fox) proteins in cancer. Nat Rev Cancer 7: 847-859. 
39. He W, Kang Y, Zhu W, Zhou B, Jiang X, Ren C and Guo W (2020). FOXF2 acts as a crucial molecule in tumours and embryonic development. Cell Death Dis 11: 424.

40. Kundu ST, Byers LA, Peng DH, Roybal JD, Diao L, Wang J, Tong P, Creighton CJ and Gibbons DL (2016). The miR-200 family and the miR-183 96 182 cluster target Foxf2 to inhibit invasion and metastasis in lung cancers. Oncogene 35: 173-186.

41. Cai J, Tian AX, Wang QS, Kong PZ, Du X, Li XQ and Feng YM (2015). FOXF2 suppresses the FOXC2mediated epithelial-mesenchymal transition and multidrug resistance of basal-like breast cancer. Cancer Lett 367: 129-137.

42. Milewski D, Pradhan A, Wang X, Cai Y, Le T, Turpin B, Kalinichenko VV and Kalin TV (2017). FoxF1 and FoxF2 transcription factors synergistically promote rhabdomyosarcoma carcinogenesis by repressing transcription of p21(Cip1) CDK inhibitor. Oncogene 36: 850-862.

\section{Figures}




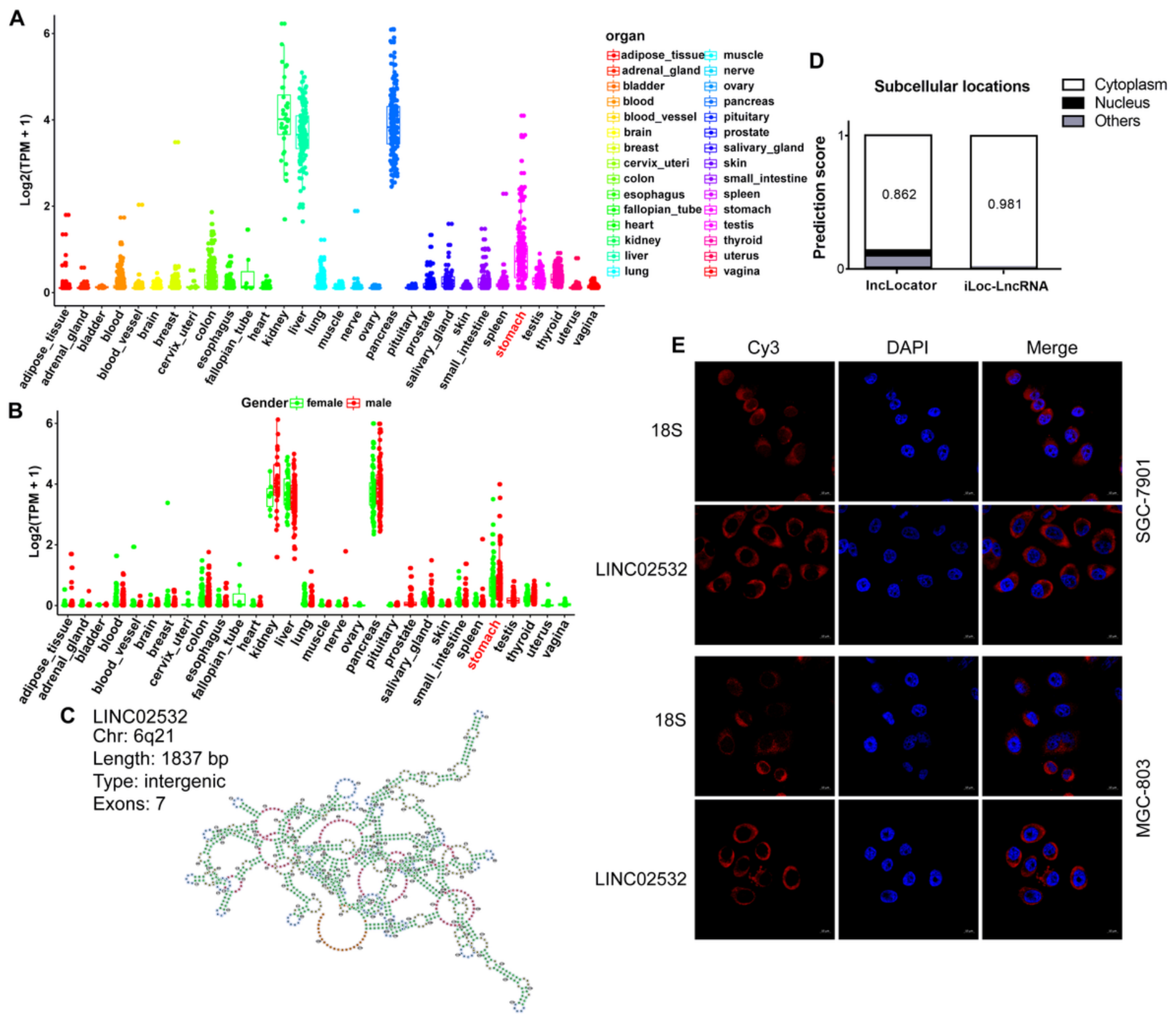

\section{Figure 1}

Expression, structure, and subcellular localization of LINC02532. (A) Expression levels of LINC02532 in multiple normal human organs from the GTEx project. (B) LINC02532 expression levels in normal tissues were not affected by differences in sex. (C) LINC02532 was identified as a non-coding RNA in the InCAR database and its secondary structure was predicted by RNAfold. (D) LINC02532 has a high probability of being located in the cytoplasm based on the IncLocator and iLoc-LncRNA prediction. (E) RNA-FISH assay indicated that LINC02532 was mainly located in the cytoplasm of SGC-7901 and MGC-803 cells. 


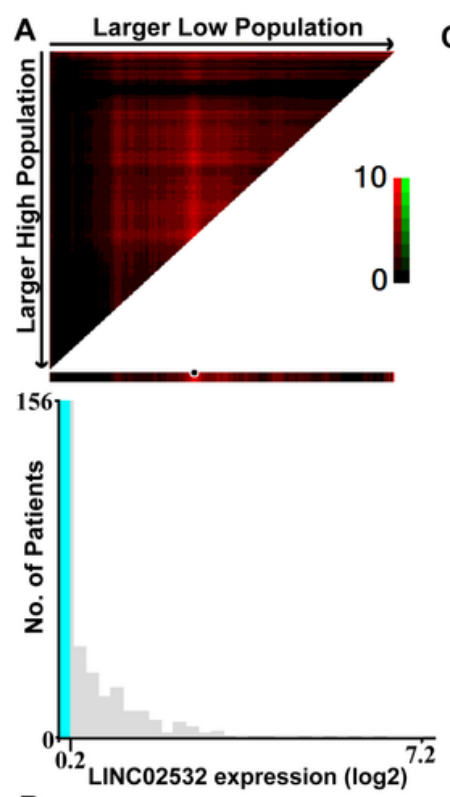

B
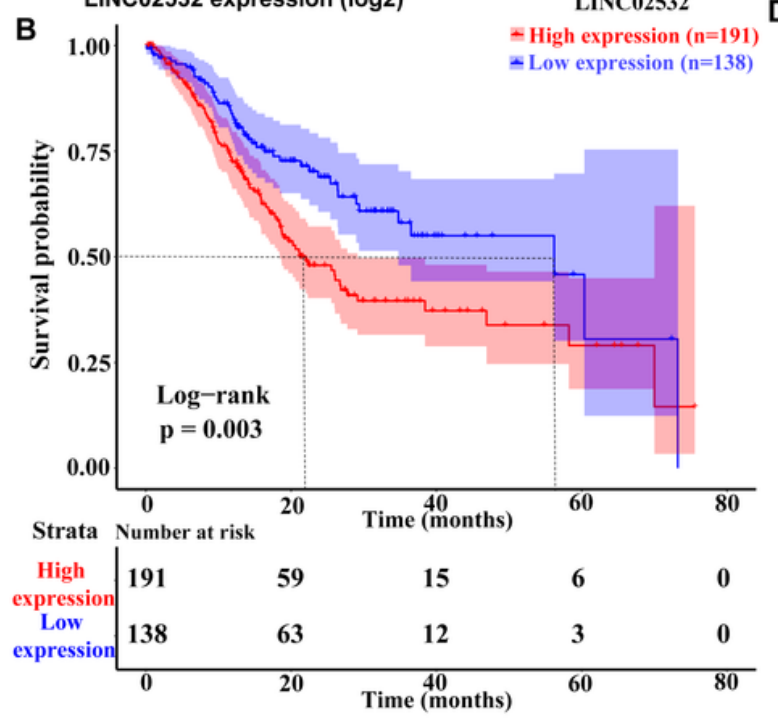
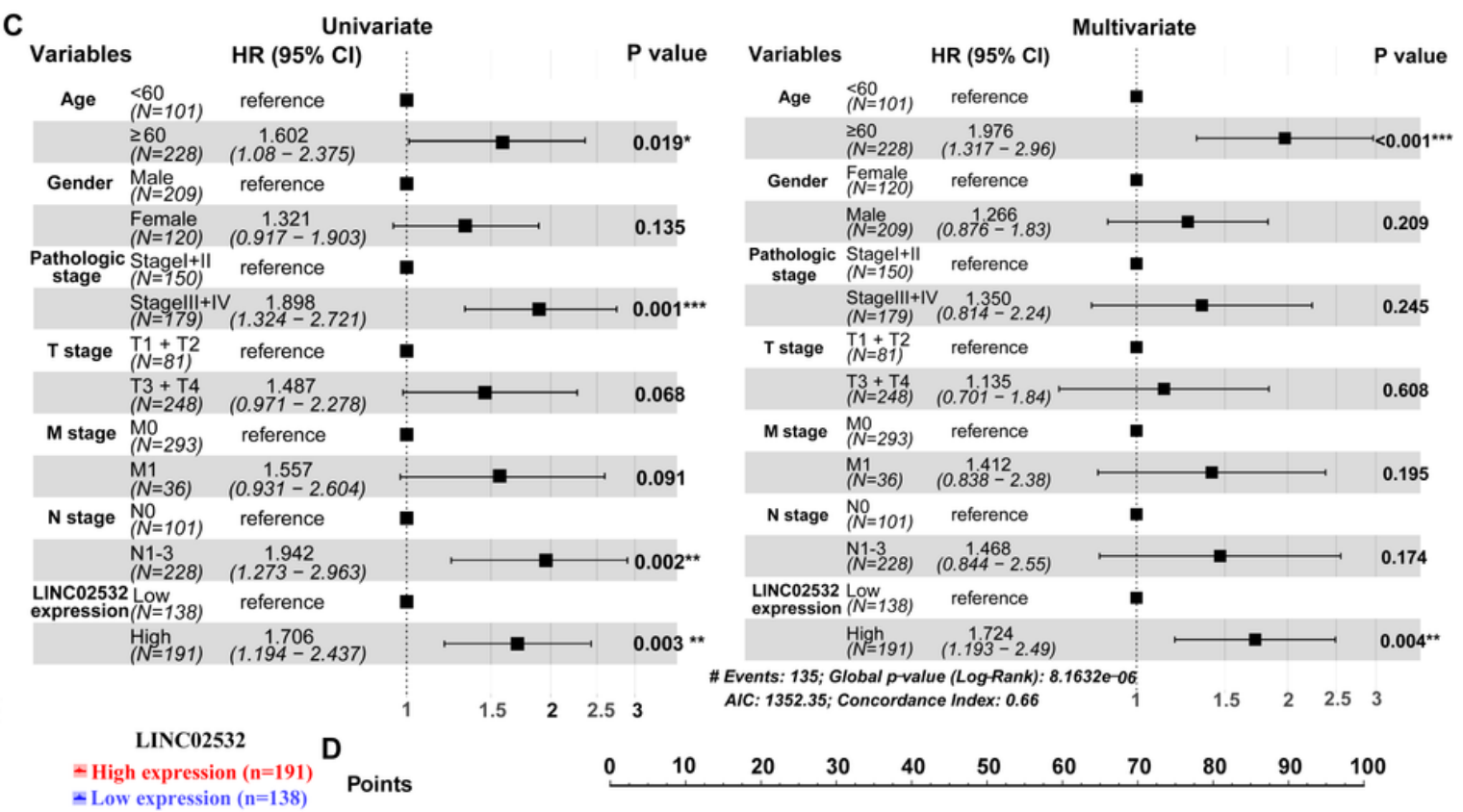

Age
M stage
N stage

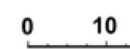

$20 \quad 30$

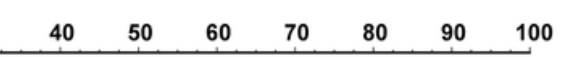

$\geq 60$

LINC02532 expression

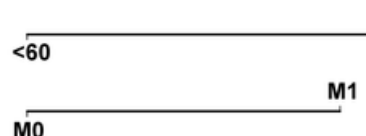

Mo

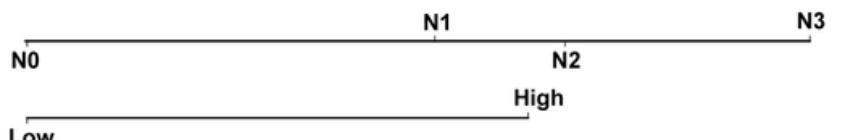

Total Points

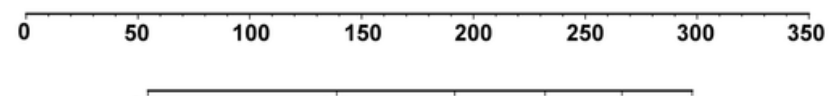

1-year survival

2-year survival

3-year survival

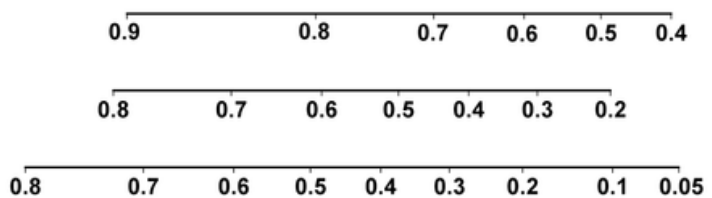

Figure 2

High LINC02532 expression is associated with poor prognosis in GC. (A) The optimal high/low LINC02532 expression cut-off value calculated by the X-tile program was 0.2 (log2 transformed). (B) Kaplan-Meier survival analysis with log-rank test showed that GC patients with higher LINC02532 expression had significantly shorter OS times than those with lower LINC02532 expression $(p=0.003)$. (C) Univariate and multivariate Cox regression analyses revealed that high LINC02532 expression was an independent prognostic factor for GC patients. (D) The nomogram constructed from the Cox regression analysis data satisfactorily predicted the 1-, 2-, and 3-year overall survival of GC patients. ${ }^{*}, \mathrm{p}<0.05 ; * \star, p$ $<0.01$, and ${ }^{* *} p<0.001$ were considered statistically significant.

\section{Figure 3}


LINC02532 overexpression significantly promoted GC cell migration and invasion in vitro. (C, D) Knockdown of LINC02532 increased the mRNA (C) and protein (D) levels of epithelial marker (E-cadherin) and decreased that of mesenchymal markers (N-cadherin, VIM, and SLUG) in SGC-7901 and MGC-803 cells. (E) LINC02532 overexpression significantly enhanced the tumour growth capacity and tumour weight in nude mice $(0.308 \pm 0.066 \mathrm{~g}$ vs. $0.120 \pm 0.031 \mathrm{~g})$. (F) LINC02532 overexpression significantly promoted the formation of mice lung metastasis nodules. (43.40 \pm 9.84 vs. $12.00 \pm 3.87)$. ${ }^{*}, p<0.05$ and **, $\mathrm{p}<0.01$ were considered statistically significant.

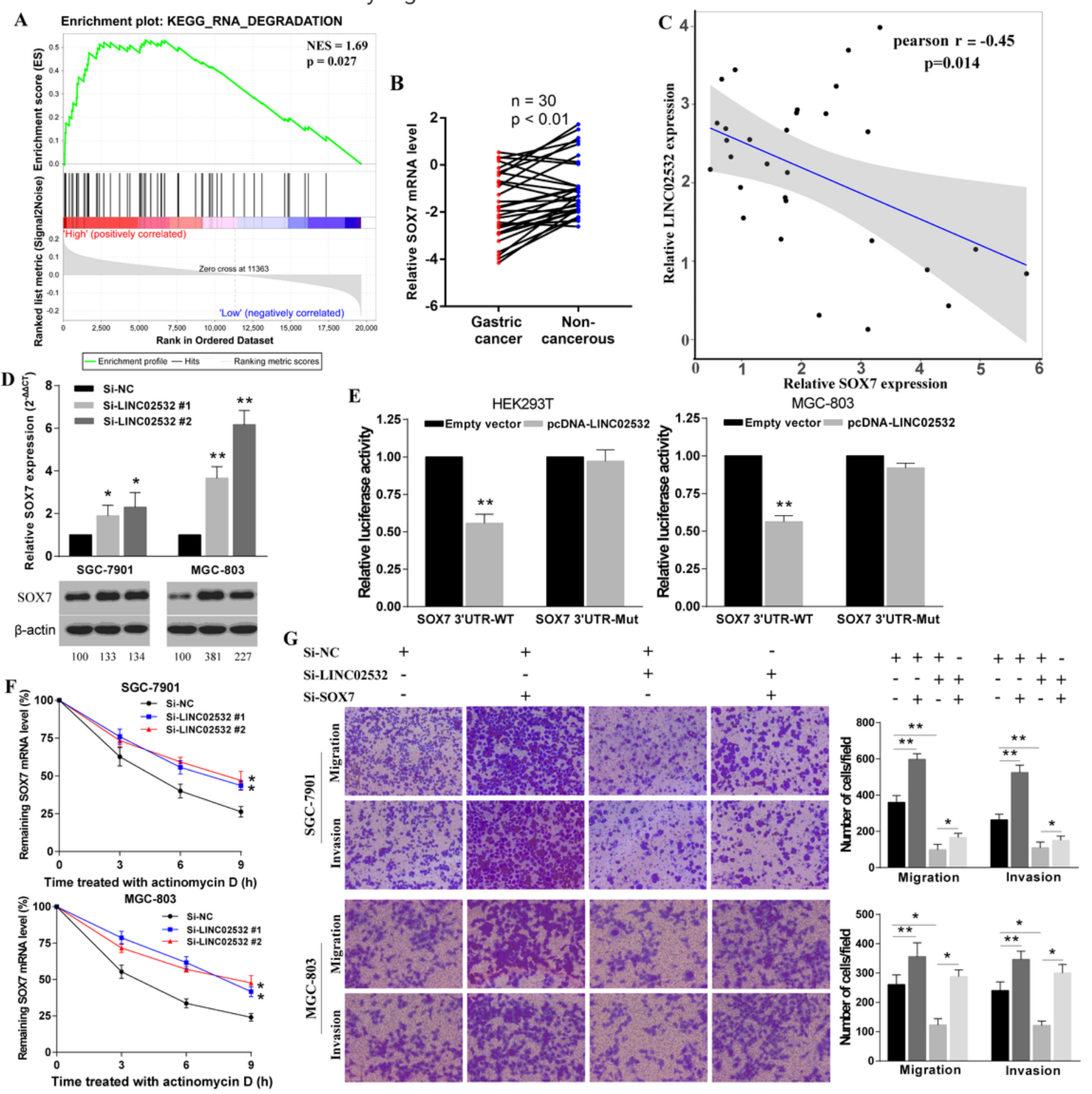

Figure 4 
LINC02532 decreases SOX7 expression through changes in mRNA stability resulting in GC cell migration and invasion. (A) The GSEA findings indicated that high LINC02532 expression was significantly positively correlated with the KEGG RNA degradation pathway (NES $=1.69, p=0.027$ ). (B) Compared with corresponding normal tissues, SOX7 mRNA levels were significantly down-regulated in GC tissues $(n=30$, $p$ < 0.01). (C) Pearson correlation analysis showed that SOX7 expression was negatively correlated with LINC02532 (Pearson $r=-0.45, p=0.0014$ ). (D) LINC02532 knockdown significantly increased the mRNA and protein expression of SOX7. (E) The dual-luciferase reporter assay indicated that LINC02532 negatively regulated SOX7 expression by binding to its 3'UTR region. (F) LINC02532 decreased the mRNA half-life of SOX7 in SGC-7901 and MGC-803 cells. (G) LINC02532 promoted GC cell migration and invasion by decreasing SOX7 expression. *, $p<0.05$ and **, $p<0.01$ were considered statistically significant.

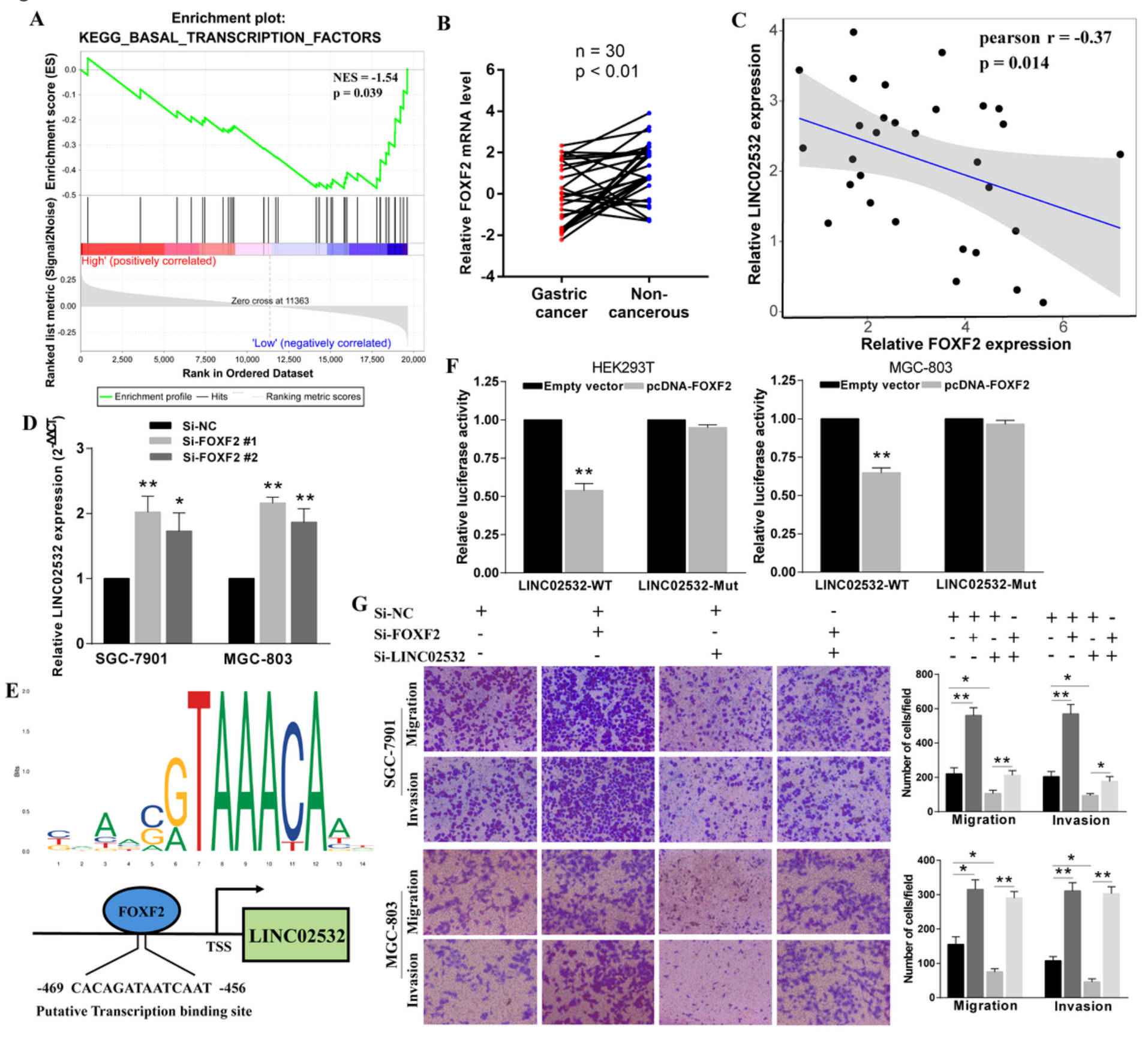


FOXF2 mediates GC cell migration and invasion through negative regulation of LINC02532 transcription. (A) The GSEA results showed that high LINC02532 expression was significantly negatively correlated with the KEGG basal transcription factors pathway. (B) FOXF2 was significantly down-regulated in GC tissues compared with the corresponding normal tissues $(n=30, p<0.01)$. (C) Pearson correlation analysis indicated that the expression of FOXF2 was negatively correlated with LINC02532 (Pearson $r=$ $-0.37, p=0.043)$. (D) LINC02532 expression was significantly up-regulated after FOXF2 expression was silenced in SGC-7901 and MGC-803 cells. (E) The binding motif of FOXF2 and a conservative FOXF2 binding site (5'-CACAGATAATCAAT-3') in the LINC02532 promoter region located at -469 to -456 bp from the TSS. (G) LINC02532 knockdown-mediated inhibition of GC cell migration and invasion was partially rescued by co-transfection with si-FOXF2. *, $p<0.05$ and **, $p<0.01$ were considered statistically significant.

\section{Image not available with this version}

\section{Figure 6}

Proposed working model showing that downregulation of FOXF2 resulted in increased expression of LINC02532, decreased stability of SOX7 mRNA and increased GC cell migration and invasion.

\section{Supplementary Files}

This is a list of supplementary files associated with this preprint. Click to download.

- SupplementaryTable.docx 